\title{
Is Active Impedance the Key to a Breakthrough for Legged Robots?
}

\section{Conference Paper}

Author(s):

Semini, Claudio; Barasuol, Victor; Boaventura, Thiago; Frigerio, Marco; Buchli, Jonas

Publication date:

2016

Permanent link:

https://doi.org/10.3929/ethz-a-010244012

Rights / license:

In Copyright - Non-Commercial Use Permitted

Originally published in:

Springer Tracts in Advanced Robotics 114, https://doi.org/10.1007/978-3-319-28872-7_1 


\title{
Is Active Impedance the Key to a Breakthrough for Legged Robots?
}

Claudio Semini, Victor Barasuol, Thiago Boaventura, Marco Frigerio, Jonas Buchli

\begin{abstract}
This work addresses the question whether active impedance control is key to a breakthrough for legged robots. In this paper, we will talk about controlling the mechanical impedance of joints and legs with a focus on stiffness and damping control. In contrast to passive elements like springs, active impedance is achieved by torque-controlled joints allowing real-time adjustment of stiffness and damping. We argue that legged robots require a high degree of versatility and flexibility to execute a wide range of assistive tasks to be truly useful to humans and thus to lead to a breakthrough. Adjustable stiffness and damping in realtime is a fundamental building block towards versatility. Experiments with our $80 \mathrm{~kg}$ hydraulic quadruped robot HyQ demonstrate that active impedance alone (thus no springs in the structure) can successfully emulate passively compliant elements during highly-dynamic locomotion tasks (running and hopping); and, that no springs are needed to protect the actuation system. Here we present results of a flying trot, also referred to as running trot. To the authors' best knowledge this is the first time a flying trot was successfully implemented on a robot without passive elements such as springs. A critical discussion on the pros and cons of active impedance concludes the paper.
\end{abstract}

Claudio Semini and Marco Frigerio

Dept. of Advanced Robotics, Istituto Italiano di Tecnologia (IIT), via Morego, 30, 16163 Genova e-mail: claudio.semini@iit.it, claudio@semini.ch and marco.frigerio@iit.it

Victor Barasuol

PPGEAS - Dept. of Automation and Systems, Federal University of Santa Catarina (UFSC), Florianpolis, SC, Brazil e-mail: victor.barasuol@ posgrad.ufsc.br

Thiago Boaventura and Jonas Buchli

Agile \& Dexterous Robotics Lab, ETH Zurich, Tannenstr. 3, 8092 Zürich e-mail: tboaventura@ethz.ch and buchlij@ethz.ch 


\section{Introduction}

Robots with arms and legs have the potential to become true assistants to humans in everyday life and might replace them for dangerous, dull and dirty tasks. While the legs will allow these robots to move with agility in any kind of terrain accessible to humans and animals, their arms will allow them to execute tasks with human dexterity. However, today's most advanced robots are still very far from this goal. In fact, the majority of today's legged robots struggle to move in even slightly rough terrain. This inability presents a stark contrast to human capabilities. The discrepancy in performance has several reasons. Historically, robot arms - and later legs were controlled with stiff position-controlled joints. Interactions with the environment had to be carefully planned in the kinematic domain since neither information about the contact dynamics and forces could easily be taken into account, nor force and torque control was available. While this may be sufficient for most tasks of today's industrial robots, an autonomous machine will never be able to obtain neither a perfect map of the environment nor a perfect robot state estimation. Thus, precise kinematic planning of footholds is not a feasible solution for tomorrow's robots that have to move and interact in challenging and dynamically changing environments. Handling collisions and non-smooth interactions has to be part of their list of specifications.

The physical laws governing interaction dynamics show that it is paramount to control also the joint torques and/or the contact forces during interactions with the environment [19], e.g. during locomotion on irregular terrain. Studies support the assumption that humans and animals are able to control joint torques thanks to antagonistically acting muscle pairs. The elasticity of the tendons in combination with muscle control allow to adjust both the passive and active joint impedance, respectively $[18,36,40]$. Active impedance for the hand or the foot is obtained by means of muscle control by co-contracting the antagonistic muscle pair [13, 10, 31]. This control naturally has a delay of few tens of milliseconds or more [15, 24]. During collisions, the passive compliance ${ }^{1}$ and damping in the tendons helps to protect the actuation system during this delay. The smaller the delay, the less passive compliance/damping is needed to prevent damage.

In the last decades, researchers have proposed several possible ways on how to more properly cope with the interaction forces with the environment. Some approaches use the passive dynamics of mechanical and pneumatic springs in the leg structure to govern the interaction dynamics (e.g. Buehler et al. [9], Raibert et al. [30]). The resonant frequency of the resulting spring-mass system is then used to achieve a resonant hopping and running motion. Pratt et al. [27] proposed the series elastic actuator (SEA) where (usually stiffer) springs are put in series to the actuator. The main purposes of the spring in a SEA is to control joint forces, absorb impact peaks and temporarily store energy. Springs are especially popular for elec-

${ }^{1}$ Compliance is the inverse of stiffness. 
trically actuated robots, as they can protect the gears ${ }^{2}$ from getting damaged during collisions and non-smooth interactions.

These springs, however, introduce passive dynamics and low-frequency resonant modes into the system and therefore have to be tuned for a certain task. While this is fine for a single-purpose machine (e.g. a robot for highly efficient running), it drastically reduces the versatility and thus usefulness of a service robot in human environments. Even the normally stiffer springs of the SEA reduce the actuator bandwidth as a result of the resonant modes, and therefore make certain tasks where a stiff and precise motion is required impossible. This topic is further elaborated in Sect. 6.

To overcome this problem researchers have been working on variable stiffness actuators (VSA) [42] that can vary the stiffness of each joint with the help of a (generally smaller) second actuator. While recent progresses in this field have increased the range of adjustable stiffnesses [41], the actuators are still bulky, complex and often cannot absorb high energy impacts due to the limited size of the springs.

Active impedance is a promising alternative that does not require any physical springs, because the required stiffness and damping is controlled by software and torque-controlled joints (e.g. impedance control [20], operational space control [25], and virtual model control [28]). Any stiffness and damping (within the limitations of the actuation and control system) can be selected in realtime either for the endeffector or for each joint independently [5]. This approach has most advantages of VSA without the above mentioned limitations. Boaventura et al. [5] present an experimental comparison study of active versus passive compliance and show that active impedance systems can emulate passive elements in the dynamic range needed for locomotion and interaction with the environment in general. The performance of the emulation is such that there is no relevant difference between the dynamic behavior of the actively controlled system and its fully passive 'template' system.

In this work we will demonstrate that active impedance can enable a legged robot to potentially execute a wide range of different tasks in natural environments and thus increase its versatility and usefulness. We will present our previous work on our torque-controlled hydraulic quadruped robot HyQ $[32,33]$ in this context. And, we will demonstrate the advantages and the potential of active impedance and torquecontrolled robots with two new experiments: a flying trot and resonant hopping. The flying trot demonstrates the robustness and performance of the impedance controller in a very demanding situation due to the high frequency impacts at the touch down moments. The resonant hopping demonstrates the flexibility and versatility of the control concept.

The major contribution of this work is the presentation of a flying trot with an $80 \mathrm{~kg}$ quadruped robot with purely impedance-controlled legs, thus without any springs in its mechanical structure. To the best knowledge of the authors no machine has achieved this before. In this paper we use the success of this experiment as an example to discuss the importance of active impedance in legged robots for real-world tasks.

${ }^{2}$ Reduction gears are required to amplify the low output torque of electric motors. 
This paper first discusses the state of the art in the field of purely impedancecontrolled legged robots and machines that successfully demonstrated a flying trot. Section 3 then introduces the active impedance controller of our quadruped robot HyQ. The control required to implement a flying trot is explained in Sect. 4; and Sect. 5 presents the experimental results of a flying trot and resonant hopping motion with variable joint stiffness. Finally, Sect. 6 discusses if active impedance can help legged robots to break through and Sect. 7 concludes the paper with final remarks.

\section{State of the Art}

We will discuss the state of the art of active impedance on legged robots and work related to experimental implementations of flying trots on quadruped robots.

\subsection{Legged Robots with Active Impedance}

In this section we will focus on legged robots with active but no passive impedance, i.e. without any physical spring in their structure. For a more general and extensive review of impedance control in robotics, including fields like haptics and manipulation, please refer to Boaventura et al. $[6,5]$. There exist only a few examples of purely impedance-controlled legs with internal torque control loop in the literature. Ott et al. [26] presented a bipedal walking robot with actuators based on the modular drives of the DLR-Lightweight-Robot-II [17]. These actuator units are based on torque-controlled electric motors with integrated joint torque sensors. The robot successfully demonstrated walking on flat ground and stairs, as well as balancing and posture control. No highly-dynamic gaits like running have been demonstrated so far. Another electrically actuated robot with purely impedance- controlled legs is the MIT cheetah robot. Seok et al. $[35,34]$ presented a quadruped robot with joint torque control, implemented with electric motors with low gear ratio (5.8:1) and current control. No springs or torque sensing elements are needed in this approach (except an elastic spine for energy storage). The robot - supported by a boom - successfully demonstrated a running gait on a treadmill and showed reliable impedance control on joint level. A similar approach was taken by Buchli et al. [8] with LittleDog that had joint level torque control based on electric motor current control. The authors showed how a feedforward torque term obtained by inverse dynamics can reduce the position gains and allow for a successful disturbance rejection of unperceived obstacles. The high gear ratio, low control bandwidth and non-robust gears, however, made it very difficult to implement well controlled dynamic gaits.

There are also a few examples of hydraulically actuated robots with only active impedance. The Sarcos humanoid robots at ATR [22], CMU [39] and more recently at USC [16] have torque controlled joints based on torque sensors. The three research groups have shown balancing and simple stepping experiments on their 
robots, but none of them has shown any more dynamic gaits. HyQ is a hydraulicallyactuated quadruped robot developed at the Istituto Italiano di Tecnologia [32, 33] with joint torque control based on torque sensors $[6,12]$. Our robot has successfully demonstrated various dynamic gaits ranging from fast walking $(2 \mathrm{~m} / \mathrm{s})$, jumping, rearing to balancing over rough and instable terrain [2]. Recent experimental studies [5] on a single leg of HyQ compared active versus passive impedance and showed that high-performance impedance controllers can satisfactorily emulate passive elements such as spring-dampers. In Sect. 5 of this paper we will show how active impedance can enable highly-dynamic and versatile locomotion.

\subsection{Robots Running with a Flying Trot}

Next, we will discuss robots that have successfully demonstrated a flying (or running) trot. Note that we include robots with active and passive impedance, SEA, etc. in this overview. Raibert's quadruped robot of the CMU and later MIT leg lab was the first quadruped robot to demonstrate a flying trot [30]. Its prismatic legs had pneumatic springs in their structure that allowed the robot to run in resonance. BigDog is a hydraulically-actuated quadruped robot [29]. In one of the online videos, this robot demonstrated a flying trot. To date, no experimental results have been published. BigDog has torque-controlled joints and springs in the last segment of its legs. We believe that a combination of active and passive impedance is used in BigDog. StarlETH is quadruped robot developed at the ETH Zurich with relatively stiff springs in series with its actuators (SEA) making it a fully torque-controlled robot [21]. This platform has recently shown trotting with short flight phases [14]. The Cheetah-cub is a $1.1 \mathrm{~kg}$ electric quadruped robot that recently demonstrated a flying trot [37]. Its legs are designed around a spring loaded pantograph mechanism.

Note that all of the above-mentioned robots have passively compliant elements (mostly springs) in their legs.

A few other robots have shown running gaits while some of their degrees of freedom are restricted by a boom or other guiding mechanism (e.g. the biped MABLE [38] (using passive compliance with active force control), KOLT [11] (springs in legs), MIT Cheetah (see Sect. 2.1), Boston Dynamics' Cheetah (no information available)), thus not fully and convincingly demonstrating the versatility required for a useful service robot.

\section{Active Impedance}

With active impedance we mean that the mechanical impedance is (actively) controlled and adjustable in software. Note that in our case we control both stiffness and damping, but did not implement inertia-shaping. 


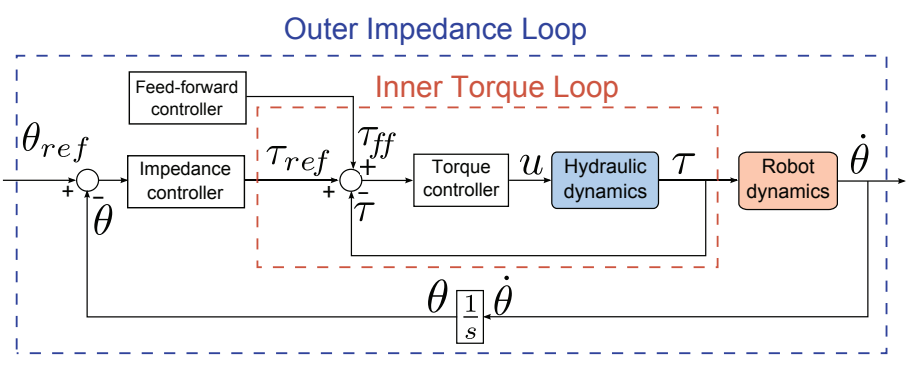

Fig. 1 Block diagram of the HyQ cascade impedance control architecture. It includes an outer impedance loop and an inner torque loop. The outer loop consists of a feedback controller and can include also a feed-forward controller such as rigid body inverse dynamics controller. The inner torque loop uses a feedback linearization approach for an increased tracking performance.

To implement active impedance on HyQ, we use a cascaded control architecture as depicted in Fig. 1. In this control scheme, an outer impedance control loop feeds back the joint angular positions and produces a torque command as output. Then, this torque command becomes the input reference for an inner torque control loop. The high performance of the inner torque controller, obtained through low-level model-based techniques $[6,12]$, was essential to successfully achieve adjustable impedance through software, without the presence of real springs.

The outer impedance loop defines the impedance characteristics of the robot, either set in joint or task space. The joint stiffness and damping can be implemented through a simple proportional derivative (PD) joint position controller. In this case, due to the presence of the inner torque loop, the proportional gain of the position control acquires units of $\mathrm{Nm} / \mathrm{rad}$, which corresponds to a rotational spring, and the derivative gain acquires the unit $\mathrm{Nms} / \mathrm{rad}$, which corresponds to a rotational damper. Therefore, by setting the proportional and derivative position gains it is possible to define the stiffness and damping of the robot joints. This joint-space impedance scheme is used for the flying trot experiments described in Sect. 5.2.

On the other hand, sometimes it might be more convenient to set the impedance at the end-effector instead of at the joints. A very intuitive way of defining a task-space stiffness and damping is through the implementation of virtual components [28]. As for the PD position controller mentioned above, these virtual components are also implemented in the impedance loop shown in Fig. 1. In HyQ, we designed a virtual spring-damper between the hip and the foot, as depicted in Fig. 6 on the left. The desired force $f$ created by these virtual components can be linear or nonlinear with respect to the stiffness, damping, and virtual prismatic leg length [6]. Once the endeffector force $f$ is calculated, it is then mapped into joint-space through the Jacobian transpose of the kinematic transform of the virtual model coordinate system to the joint coordinate system. The use of the virtual prismatic leg is also a simple way of actively implementing the well-known spring loaded inverted pendulum (SLIP) model [4], which is a useful abstraction that describes the spring-like behaviour found in human and animal running and walking. This task-space impedance controller is employed in this paper in Sect. 5.3, where the stiffness of the linear spring is changed on the fly to create a resonant hopping with $\mathrm{HyQ}$. 
In addition, the inner torque controller permits a straightforward implementation of high-level model-based control techniques, such as rigid body inverse dynamics, and gravity compensation. The output torques from the above mentioned techniques can be easily added as a feed-forward torque to the torque reference command from the outer loop, as shown in Fig. 1. Some of these model-based techniques provide very convenient capabilities for performing robust locomotion in unstructured and partially unknown environments [8]. Essentially, such control methods permit lowering the position gains (hence the system's stiffness) without giving up on position tracking performance.

\section{Flying Trot Motion Generation and Control}

A trot is a gait in which diagonal leg pairs move simultaneously, alternating with the other pair of legs. A flying trot (or running trot) is a special case characterized by a ballistic body motion, i.e., by a period in which there are no legs in contact with the ground. The body flight phase depends on the ratio between the time that a leg stays in contact with the ground (the stance phase) and the time that a leg takes to swing to the next foothold (the swing phase). This ratio is called Duty Factor, hereafter defined as $D_{f}$, and varies between 0 and 1. During trotting, if all the legs have a duty factor of less than 0.5 (i.e. swing phase longer than stance phase) then the body undergoes a flight phase for a certain time fraction of the gait cycle.

A comprehensive locomotion control framework is required to make a robot perform a stable flying trot. This control framework needs to integrate appropriate trajectory generation and body motion control in a closed loop fashion. Our recently presented Reactive Control Framework (RCF) [2] implements these aspects and we adapted it to achieve a flying trot with HyQ. The RCF integrates the basic components for robot motion generation and robot motion control. No information about the environment, such as terrain surface level or obstacles, is required to achieve a basic robust (reactive) locomotion behavior.

Next, we will highlight some of the important features of the RCF in relation to the generation of a flying trot: the generated profile for the feet trajectories; the trajectory generator parameters; and how we choose such parameters to achieve a flying trot.

The generation of the reference trajectories for the feet is loosely inspired by the Central Pattern Generators (CPGs) of animals [23], with the advantage of having intuitive parameters such as step length and step height. Ellipse-shaped trajectories (called primitives) are generated by a network of four non-linear oscillators, whose state represents the Cartesian coordinates of each foot [3], as depicted in Fig. 2 on the left. The oscillator parameters that define the aspect ratio of the ellipse are directly related to the step length $L_{s}$ and the step height $H_{s}$. Each oscillator has an angular frequency $w_{s}$, associated to the corresponding leg step frequency $f_{s} ; w_{s}$ might be different for the stance and swing phases, to achieve a duty factor different from 0.5 . Non-linear filters are coupled to the output of the network of oscillators to 
reshape the elliptical trajectories to semi-elliptical ones, to make the robot capable of adapting to the actual terrain profile. The non-linear filters reshape the primitive's trajectories according to an estimation of the foot position at touchdown; this information is either predefined, when the surface is well known, or computed from sensory information (for example using force sensors). The shape of the adapted trajectories are illustrated in Fig. 2 on the right.

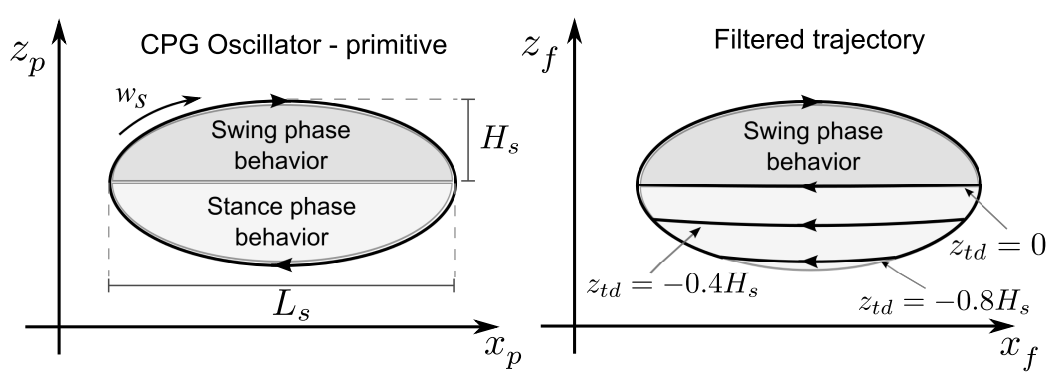

Fig. 2 The foot trajectory generated by the CPG oscillator (on the left) and the trajectory modulated by the non-linear filter (on the right). $z_{p}$ and $x_{p}$ are the reference coordinates of the primitive's trajectory, while $z_{f}$ and $x_{f}$ are the filtered references sent to the joint controller. $z_{t d}$ is the filter parameter which determines where the original elliptic trajectory has to be interrupted. (Figure modified from Barasuol et al. [2])

The step depth parameter $z_{t d}$ affects the reshaping of the trajectory by determining at which height the ellipse has to be interrupted, as depicted in Fig. 2 on the right. The desired robot forward velocity $V_{f}$ determines the relative velocity of the foot with respect to the robot trunk, which is imposed during the rectified part of the semi-ellipse (i.e. during the stance phase). If a terrain map is available the swing-tostance transition can be planned in advance, reducing the impact forces. On the other hand, the feet trajectories can be dynamically adjusted even if the robot is walking blindly, e.g. by using feedback from the foot or joint force sensing, see [2]. This feature makes locomotion more robust also with respect to poor state estimation.

In this paper we show experiments performed on flat ground. We consider the flat ground as a well-known surface and, therefore, we assume $z_{t d}=0$ for all the legs. With $z_{t d}=0$ the shape of the primitives becomes a half-ellipse.

During a flying trot the most important parameters are the step length $L_{s}$, the duty factor $D_{f}$, the desired forward velocity $V_{f}$ and the step frequency $f_{s}$. In the RCF approach all these parameters can be independently modulated. To achieve a stable spring-mass bouncing motion of the robot's centre of mass (COM), the robot's motion during the stance period needs to match the system's resonant frequency (defined by the robot's mass and leg stiffness). Selecting a proper duty factor and step frequency allows us to obtain a stance phase that matches the natural resonance period. Since $D_{f}$ and $f_{s}$ are then defined, choosing a desired forward velocity $V_{f}$ consequently determines the value of the step length $L_{S}$.

In our flying trot we explore the independent parameter modulation capability of the RCF approach to generate a variable swing velocity of the leg. The idea is to 
move the leg faster in regions where there is a low risk of impact with obstacles, while slowing it down in proximity of the expected touchdown regions, to reduce the impact forces. We obtain this leg behavior by modulating the angular frequency of the primitives according to the collision-free region and the unknown touch-down region, without affecting the total swing period. See Fig. 3(a).

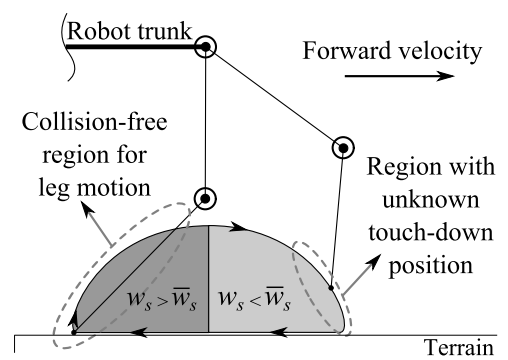

(a)

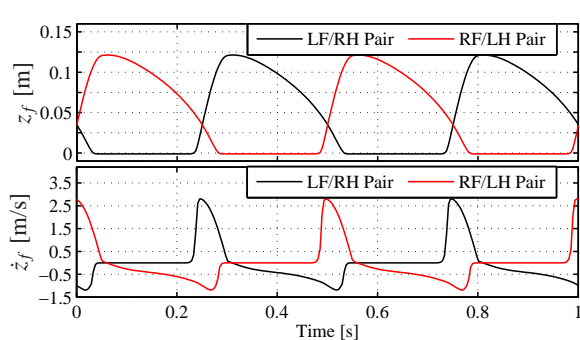

(b)

Fig. 3 Modulation of the angular frequency $w_{s}$. (a) In the collision-free region the angular frequency $w_{s}$ is greater than the average angular frequency $\bar{w}_{s}$ of the swing phase. In the unknown touch-down region $w_{s}$ is smaller than $\bar{w}_{s}$. (b) The plot shows the foot's relative position $z_{f}$ (step height) and the corresponding velocity $\dot{z}_{f}$ references for each pair of diagonal legs (LeftFront/Right-Hind and Right-Front/Left-Hind legs). The swing period in the collision-free region is chosen to be half of the swing period in the unknown touch-down region. The duty factor is 0.45 , the desired forward velocity is $1 \mathrm{~m} / \mathrm{s}$, the step frequency is $2 \mathrm{~Hz}$ and the step height is $0.12 \mathrm{~m}$.

Figure 3(b) shows an example of Cartesian references for a flying trot run at 1 $\mathrm{m} / \mathrm{s}$ when the swing period in the collision-free region is chosen to be half of the swing period in the unknown touch-down region.

\section{Experimental Results}

We performed a series of experiments with our quadruped robot HyQ that uses only active (and no passive) impedance. After a description of the platform, we will present the results of a successful flying trot experiment and a resonant hopping. Both examples illustrate the advantages and potentials of active impedance for legged robots.

\subsection{Experimental Platform $\mathrm{Hy} Q$}

The platform used for these experiments is HyQ, a quadruped robot with hydraulically and electrically actuated joints [32,33]. The machine weighs $80 \mathrm{~kg}$, is roughly 1 meter long and has a leg length of $0.78 \mathrm{~m}$ with fully-extended legs. All of its 12 de- 
grees of freedom (DOF) are torque-controlled joints: The hip abduction/adduction joints are driven by DC brushless motors with strain-gauge based torque sensors for torque control [12]. All 8 joints in the sagittal plane (hip and knee flexion/extension) are actuated by hydraulic cylinders connected to load cells for force measurement. High-performance servovalves enable joint-level torque control with excellent tracking [6] that led to the implementation of active impedance as described in Sect. 3. Note, that besides a thin rubber layer at the feet, there are no passive stiffness/damping elements (e.g. springs) present anywhere in the robot's leg structure.

Since $2011 \mathrm{HyQ}$ has demonstrated a wide range of static and dynamic motions such as a crawl gait, stair climbing, walking trot over flat, inclined and rough terrain (indoors and outdoors), squat jumps, rearing, balancing under disturbances and step reflexes.

\subsection{Flying Trot Experiment}

We conducted several experiments of a flying trot with HyQ based on the approach presented in Sect. 4. Figure 4 shows a picture sequence of one of these experiments to illustrate the flight phases (right hand side frames) between the stance phases of the two diagonal leg pairs. A link to a video of this experiment can be found at [1].
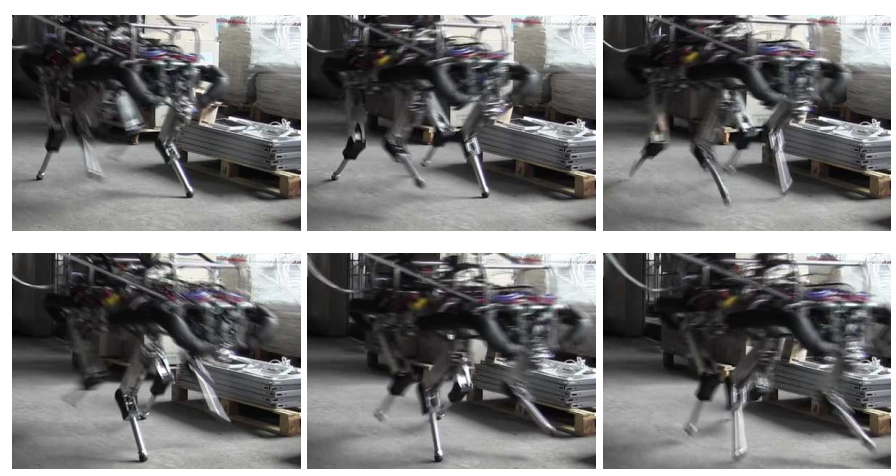

Fig. 4 Picture sequence of the flying trot experiment with the HyQ robot, which shows the flight phase achieved by setting the duty factor at 0.45 . The time between each frame is $80 \mathrm{~ms}$, and the whole sequence represents $400 \mathrm{~ms}$ of the actual experiment.

Figure 5 shows the knee joint torque plots of the four legs and the vertical ground reaction forces. The duty factor during this experiment was set to 0.45 , the step height $0.12 \mathrm{~m}$, forward velocity $1.3 \mathrm{~m} / \mathrm{s}$, step length $0.28 \mathrm{~m}$ and the joint-level active stiffness $300 \mathrm{Nm} / \mathrm{rad}$ for the hip and knee flexion/extension joints. Note that the joint torques of all four legs stay inside the maximum torque limits ${ }^{3}$ of $181 \mathrm{Nm}$

\footnotetext{
${ }^{3}$ Note that we recently increased the hydraulic system pressure of the HyQ robot to $20 \mathrm{MPa}$, increasing the maximum torque of the hip and knee flexion/extension joints to $181 \mathrm{Nm}$.
} 
demonstrating that active compliance can successfully absorb the high impacts during the running and cope with these collisions. The plots also show that the joint torques and ground reaction forces go to zero between the stance phases of the diagonal leg pairs. This illustrates that the robot was indeed in flight phases. To the best of our knowledge no other robot has successfully shown a robust flying trot with active impedance only, i.e. without passive elements such as springs in its legs.

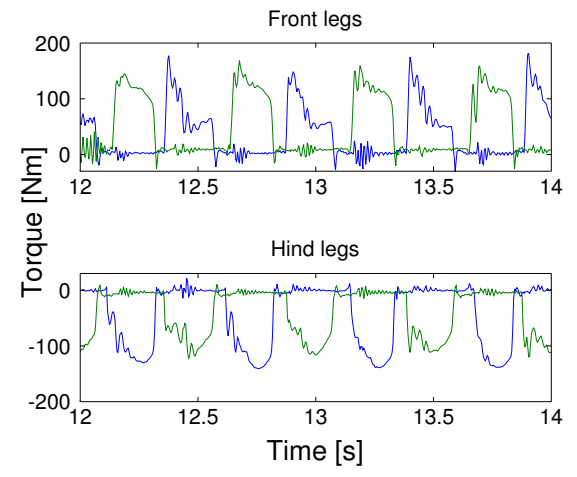

(a) Torque at the knee joints

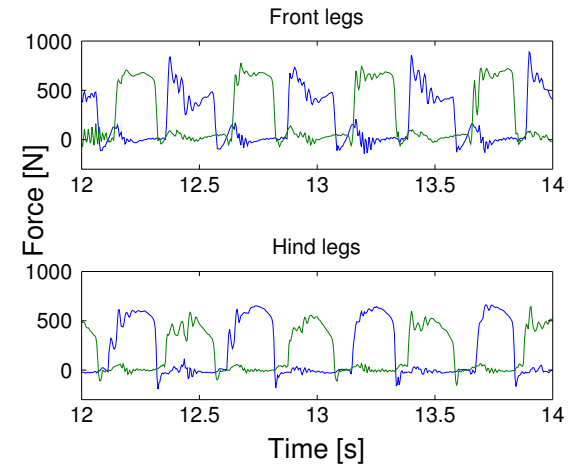

(b) Vertical ground reaction forces

Fig. 5 Force profiles during the flying trot experiment; the blue lines refer to the left legs, the green ones to the right legs. (a) This plot shows the torques at the four knee joints of the robot; the short intervals during which all the torques are close to zero are due to the flight phase. (b) This plot illustrates the ground reaction forces during the same time interval, estimated from the torques at the knees and hips with the transpose of the Jacobian.

\subsection{Resonant Hopping Experiment}

In this section we show HyQ's ability of changing the virtual spring stiffness on the fly to achieve a resonant hopping motion. For doing so, we implemented a virtual linear spring-damper for all four legs of HyQ as shown in Fig. 6 on the left. The length of the virtual linear springs $(l=0.58 \mathrm{~m})$ is varied sinusoidally $(\delta l=0.05 \mathrm{~m})$ at a constant frequency of $1.6 \mathrm{~Hz}$. During the experiment, the stiffness of the virtual springs is linearly changed from $K=2000$ to $K=5000 \mathrm{~N} / \mathrm{m}$.

As shown in Fig. 6, after $1 \mathrm{~s}$ the spring stiffness starts to increase and, consequently, the amplitude of the ground reaction force oscillations grows due to resonant effects. We show the ground reaction force for the left front (LF) leg in the first plot. When the stiffness and thus spring-mass system resonates with the frequency of the sinusoidal spring length excitation, the robot starts to hop and the ground reaction forces go to zero during flight phase (all four legs in the air). The resonance peak occurs at about $10 \mathrm{~s}$, when the stiffness is around $3800 \mathrm{~N} / \mathrm{m}$.

This example shows how active impedance allows to adjust the dynamics of the system, thus creating a big potential for new control methods for legged robots. 

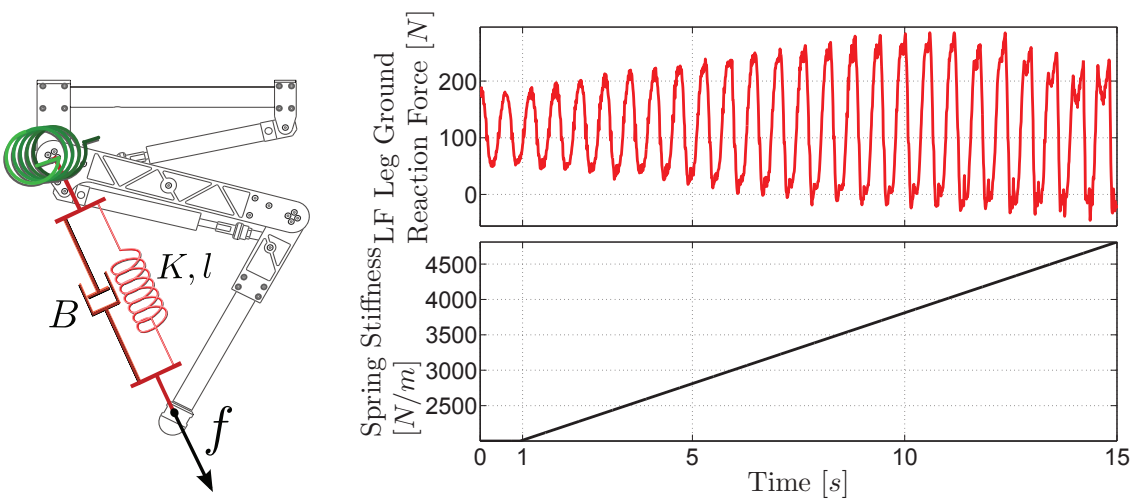

Fig. 6 Experimental results of resonant hopping: left: HyQ virtual elements: a spring-damper connects the hip to the foot (red elements), creating a prismatic virtual leg. In the hip joint, a simple joint-space position PD control can be seen as a rotational spring-damper (green element). right: We implemented a hopping motion by exciting the HyQ robot in a resonant way by varying the virtual legs stiffness. The top plot shows the ground reaction force for the left front (LF) leg, which reaches zero after around $9 \mathrm{~s}$ demonstrating a presence of a flight phase. The bottom plot presents the linear change in stiffness applied to the legs.

\section{Discussion}

An important contribution of this work is to discuss the initial question Is Active Impedance the Key to a Breakthrough for Legged Robots? To this end, this section will first provide possible reasons why legged robots are still far from a breakthrough. We will then discuss why springs are currently not ideal to use, and mention the pro and cons of active impedance. Finally, we will propose important future topics of research that will help legged robots become a reality in every-day life.

As mentioned in the introduction, despite decades of research on legged locomotion, today's robots are still far from being able to move in human environments. Two of the main requirements for such robots are (1) the ability to cope with collisions and non-smooth interactions, since they cannot be avoided in such environments; and (2) the versatility of such machines to execute a wide range of tasks to become truly useful assistants. Very few examples of robot designs and their associated control framework meet these two requirements.

Springs are often used to meet the first requirement. However, springs are not an ideal solution to meet the second requirement for the following reasons. A truly versatile robot should be able to execute tasks ranging from a precise and careful manipulation of a delicate object, to locomotion in environments with unperceived obstacles where a soft interaction but also fast reflex motions are required. While some tasks require very precisely controlled joints, others need compliant behavior, yet others require very fast joint motions as reaction to an external perturbation, e.g. when being pushed or for safety stops. Precise motions at any speed require 
either a very good model ${ }^{4}$ of the robot and possibly the environment or high gain (i.e. 'stiff') control ${ }^{5}$. In addition, if fast motions are required, as a reaction to an unforeseen event (side step to keep balance, step reflex or stopping a robot arm in front of a person) a very high actuation bandwidth is required. Compliant behavior as reaction to an unforeseen perturbation requires low output impedance and is in contradiction to a quick controlled movement without using a model. It fundamentally limits the ability of a quick stop or a sudden reactive movement. Therefore, a compliant robot (or human) needs to have the required bandwidth and high gain control available to be robust in such situations (e.g. a safety stop of a human arm requires immediate stiffening up). In case of a SEA the spring stiffness fundamentally limits the control bandwidth and a trade-off has been fixed at design time. As mentioned in the introduction, VSA might be a possible solution to this problem, however the technology has (still) several limitations. For a VSA, the ability of a quick stop is fundamentally limited by the (usually slow) adaptation of the stiffness. In case of an active impedance system, the only limitations stem from sensing and actuation delays (actuator physics, data acquisition, data processing), which are, to a large extent, design parameters.

To sum up, a versatile robot needs to be able to control its joint stiffness in a wide range. Springs in the structure of a robot including the stiff springs of SEA reduce the maximum joint stiffness and control bandwidth; and thus the robot's versatility. We argue that legged robots with active impedance, while certainly not the only solution, are a promising solution that meet both of the above mentioned requirements. Importantly, they are implementable with today's available technology thus putting versatile service robots within immediate reach.

Active impedance has several advantages when compared to passive springs and dampers. With today's advances in actuator, control and computer technology a wide range of stable stiffness and damping values can be emulated [5], which leads to more versatile robots. These values can be adjusted in real-time to swiftly adapt to changing conditions in the environment or task. Furthermore, robots with active impedance can take advantage of any programmable type of impedance (e.g. exponential springs, nonlinear dampers, muscle-model-based springs, etc.) [6]. A potential drawback of active compliance is low energy efficiency, as no energy can be stored due to a lack of physically compliant elements. Despite this disadvantage we do not consider it as a major problem for the following reasons: On one hand, new methods of high-density energy storage are currently investigated in various research fields. New compact energy sources will eventually be able to power legged robots for entire days [7]. On the other hand, other ways of energy recovery such as energy regenerative electronics for electric motors have recently been proposed for joints with active impedance [35]. Furthermore, passively compliant elements are only really able to increase energy efficiency of a robot during repetitive motions,

\footnotetext{
${ }^{4}$ Note that the fact that models are required for good performance does not address the question where the model comes from. For robots it can sometimes be derived from CAD data, sometimes must be estimated/learned. For humans models are typically acquired by learning.

${ }^{5}$ It is worthwhile discussing these issues in the control theoretic notions of nominal behavior and disturbance reaction.
} 
such as walking, running, scrubbing etc. if the motion frequency is around the resonant frequency of the system. For an in-depth discussion of the pro \& cons of active versus passive compliance we refer the interested reader to [5].

For humans we see this trade-off in the example of Oscar Pistorious, a belowknee amputee who has won several medals in sprint running. He uses two carbonfibre curve-shaped springs as foot prosthesis that allow him to run fast and efficiently. After the races and trainings however he wears normal, stiff prosthesis. It is therefore a good example of the limited versatility imposed by springs.

Now that we understand the limitations introduced by springs we can rethink and adjust future research agendas to focus on the important topics that will lead to a faster breakthrough of legged robots into everyday life. First of all, torque-controlled robots open up a wide range of control methods besides active impedance, e.g. model-based control of rigid body dynamics (gravity compensation, inverse dynamics, etc.) and control of contact forces. These are all methods that will lead to improved manipulation and locomotion skills in human environments. Additionally, research is required into optimal selection of stiffness trajectories for a large range of tasks. Investigations into how to build more compact and less complex VSA with fast stiffness adjustment are important because they might eventually be useful to save energy during repetitive motions. Questions regarding the safety and reliability of active impedance systems were not discussed in this work due to lack of space, but they are important topics that need to be investigated. Last but not least more research into energy efficient active impedance systems is required.

\section{Conclusions}

We have shown, to the best of our knowledge, for the first time how a legged robot with active impedance only (i.e. without springs) can execute highly dynamic tasks that involve large and impulsive impact forces, such as running and hopping. Our experiments presented here and elsewhere [5] show that it is possible to achieve the same behavior with a fully actively controlled system as with passive systems. Active impedance offers the additional advantage of versatility and flexibility, allowing to specify the most suitable dynamic behavior on the fly. The data shown indicates that the argument of active systems being too slow to control does not hold for the dynamic range that is used for highly dynamic locomotion and interaction tasks on time-, force- and length-scales typical for humans. We consider this approach fundamental to the breakthrough of versatile robotic assistants with arms and legs and we have demonstrated that the required control performance is achievable.

Acknowledgements This research has been funded by the Fondazione Istituto Italiano di Tecnologia. The authors would like to thank CAPES for the scholarship granted to V. Barasuol (Grant Procs. 6463-11-8). T. Boaventura is partially funded through the EU Project BALANCE (Grant 601003 of the EU FP7 program). J. Buchli is supported by a Swiss National Science Foundation professorship. The authors would like to thank also the other members of the Dynamic Legged Systems Lab that contributed to the success of this project: M. Focchi, I. Havoutis, S. Bazeille, J. Goldsmith, H. Khan, B.Rehman, and our team of technicians. 


\section{References}

[1] online video: HyQ Robot: Flying Trot with Active Compliance: http://www.iit.it/hyq or http://www.youtube.com/watch?v=27lxHMp9LIA.

[2] V. Barasuol, J. Buchli, C. Semini, M. Frigerio, E. R. De Pieri, and D. G. Caldwell. A reactive controller framework for quadrupedal locomotion on challenging terrain. In IEEE Int. Conf. on Robotics and Automation (ICRA), 2013.

[3] V. Barasuol, V. J. De Negri, and E. R. De Pieri. WCPG: a central pattern generator for legged robots based on workspaceintentions. In Proceedings of the ASME Dynamic System and Control Conference (DSCC), pages 111-114, 2011.

[4] R. Blickhan. The spring-mass model for running and hopping. Biomechanics, 22:1217-1227, 1989.

[5] T. Boaventura, G. A. Medrano-Cerda, C. Semini, J. Buchli, and D. G. Caldwell. Stability and performance of the compliance controller of the quadruped robot hyq. In IEEE/RSJ International Conference on Intelligent Robots and Systems (IROS), 2013.

[6] T. Boaventura, C. Semini, J. Buchli, M. Frigerio, M. Focchi, and D. G. Caldwell. Dynamic torque control of a hydraulic quadruped robot. In IEEE International Conference in Robotics and Automation (ICRA), pages 1889-1894, 2012.

[7] P. G. Bruce, S. A. Freunberger, L. J. Hardwick, and J.-M. Tarascon. Li-O2 and Li-S batteries with high energy storage. Nature Materials, 11:19 - 29, 2012.

[8] J. Buchli, M. Kalakrishnan, M. Mistry, P. Pastor, and S. Schaal. Compliant quadruped locomotion over rough terrain. In IEEE/RSJ International Conference on Intelligent Robots and Systems (IROS), pages 814-820, 2009.

[9] M. Buehler, R. Battaglia, R. Cocosco, G. Hawker, J. Sarkis, and K. Yamazaki. SCOUT: a simple quadruped that walks, climbs, and runs. In International Conference on Robotics and Automation (ICRA), volume 2, pages 1707-1712, 1998.

[10] E. Burdet, R. Osu, D. Franklin, T. Milner, and K. M. The central nervous system stabilizes unstable dynamics by learning optimal impedance. Nature, 414(6862):446-9, 2001.

[11] J. Estremera and K. J. Waldron. Thrust control, stabilization and energetics of a quadruped running robot. International Journal of Robotics Research, 27:1135-1151, 2008.

[12] M. Focchi, T. Boaventura, C. Semini, M. Frigerio, J. Buchli, and D. G. Caldwell. Torquecontrol based compliant actuation of a quadruped robot. In Proc. of the 12th IEEE Int. Workshop on Advanced Motion Control (AMC), 2012.

[13] D. Franklin, E. Burdet, R. Osu, M. Kawato, and T. Milner. Functional significance of stiffness in adaptation of multijoint arm movements to stable and unstable dynamics. Exp Brain Research, 151:145-157, 2003.

[14] C. Gehring, S. Coros, M. Hutter, M. Bloesch, M. Hoepflinger, and R. Siegwart. Control of dynamic gaits for a quadrupedal robot. In IEEE International Conference on Robotics and Automation (ICRA), 2013.

[15] H. Geyer and H. Herr. A muscle-reflex model that encodes principles of legged mechanics produces human walking dynamics and muscle activities. IEEE Trans Neural Syst Rehabil Eng, 18(3):263-273, 2010.

[16] A. Herzog, L. Righetti, F. Grimminger, P. Pastor, and S. Schaal. Momentum-based balance control for torque-controlled humanoids. In arXiv preprint arXiv:1305.2042, 2013.

[17] G. Hirzinger, A. Albu-Schaffer, M. Hahnle, I. Schaefer, and N. Sporer. On a new generation of torque controlled light-weight robots. In IEEE International Conference on Robotics and Automation (ICRA), volume 4, pages 3356-3363 vol.4, 2001.

[18] N. Hogan. Adaptive control of mechanical impedance by coactivation of antagonist muscles. IEEE Trans Automatic Control, 29:681-690, 1984.

[19] N. Hogan. Impedance control: An approach to manipulation: Part I - Theory. ASME Journal of Dynamic Systems, Measurement, and Control, 107:1-7, 1985.

[20] N. Hogan. Impedance control: An approach to manipulation: Part II - Implementation. ASME Journal of Dynamic Systems, Measurement, and Control, 107:8-16, 1985. 
[21] M. Hutter, C. Gehring, M. Bloesch, M. Hoepflinger, C. D. Remy, and R. Siegwart. Starleth: A compliant quadrupedal robot for fast, efficient, and versatile locomotion. In Int. Conf. on Climbing and Walking Robots (CLAWAR), 2012.

[22] S. Hyon, J. Hale, and G. Cheng. Full-body compliant human-humanoid interaction: Balancing in the presence of unknown external forces. IEEE Transactions on Robotics, 23(5):884898, 2007.

[23] A. J. Ijspeert. Central pattern generators for locomotion control in animals and robots: a review. Neural Networks, 21(4):642-653, 2008.

[24] E. Kandel, J. Schwartz, and T. Jessell. Principles of Neural Science. McGraw-Hill Medical, 4th edition, 2000.

[25] O. Khatib. A unified approach for motion and force control of robot manipulators: The operational space formulation. Robotics and Automation, IEEE Journal of, 3(1):43-53, 1987.

[26] C. Ott, O. Eiberger, J. Englsberger, M. A. Roa, and A. Albu-Schaeffer. Hardware and control concept for an experimental bipedal robot with joint torque sensors. Journal of the Robotics Society of Japan, 30(4):378-382, 2012.

[27] G. Pratt and M. Williamson. Series elastic actuators. In IEEE International Conference on Intelligent Robots and Systems (IROS), 1995.

[28] J. Pratt, C. Chew, A. Torres, P. Dilworth, and G. Pratt. Virtual model control: An intuitive approach for bipedal locomotion. IJRR, 20(2):129-143, 2001.

[29] M. Raibert, K. Blankespoor, G. Nelson, R. Playter, and the BigDog Team. Bigdog, the rough-terrain quadruped robot. In Proceedings of the 17th World Congress The International Federation of Automatic Control (IFAC), 2008.

[30] M. H. Raibert. Legged Robots That Balance. The MIT Press, 1986.

[31] L. Selen, D. Franklin, and D. Wolpert. Impedance control reduces instability that arises from motor noise. J Neurosci, 29(40):12606-16, 2009.

[32] C. Semini. HyQ - Design and Development of a Hydraulically Actuated Quadruped Robot. $\mathrm{PhD}$ thesis, Istituto Italiano di Tecnologia (IIT), 2010.

[33] C. Semini, N. G. Tsagarakis, E. Guglielmino, M. Focchi, F. Cannella, and D. G. Caldwell. Design of HyQ - a hydraulically and electrically actuated quadruped robot. Journal of Systems and Control Engineering, 225(6):831-849, 2011.

[34] S. Seok, A. Wang, M. Y. M. Chuah, D. Otten, J. Lang, and S. Kim. Design principles for highly efficient quadrupeds and implementation on the mit cheetah robot. In IEEE Int. Conf. on Robotics and Automation (ICRA), 2013.

[35] S. Seok, A. Wang, D. Otten, and S. Kim. Actuator design for high force proprioceptive control in fast legged locomotion. In IEEE/RSJ Intelligent Robots and Systems (IROS), pages 1970-1975, 2012.

[36] R. Shadmer and M. Arbib. A mathematical analysis of the force-stiffness characteristics of muscles in control of a single joint system. Biol. Cybern., 66:463-477, 1992.

[37] A. Sproewitz, A. Tuleu, M. Vespignani, M. Ajallooeian, E. Badri, and A. Ijspeert. Towards Dynamic Trot Gait Locomotion-Design, Control and Experiments with Cheetah-cub, a Compliant Quadruped Robot. IJRR, 32(8):933 - 951, 2013.

[38] K. Sreenath, H.-W. Park, and J. W. Grizzle. Design and experimental implementation of a compliant hybrid zero dynamics controller with active force control for running on mabel. In IEEE International Conference in Robotics and Automation (ICRA), 2012.

[39] B. Stephens and C. Atkeson. Modeling and control of periodic humanoid balance using the linear biped model. In IEEE-RAS International Conference on Humanoid Robots (Humanoids), 2010.

[40] K. Tee, D. Franklin, M. Kawato, T. Milner, and B. E. Concurrent adaptation of force and impedance in the redundant muscle system. Biol. Cybern., 120(1):31-44, 2009.

[41] N. Tsagarakis, I. Sardellitti, and D. Caldwell. A new variable stiffness actuator (compactvsa): Design and modelling. In IEEE/RSJ International Conference on Intelligent Robots and Systems (IROS), pages 378-383, 2011.

[42] B. Vanderborght et al. Variable impedance actuators: a review. Robotics and Autonomous Systems, 2013. 